ISSN: 0514-7336 — ISSN electrónico: 2386-3943

DOI: https://doi.org/10.14201/zephyrus2021876381

\title{
LA VISIBILIDAD COMO FACTOR DE LOCALIZACIÓN EN LOS MEGALITOS DEL SUR DE GALICIA
}

\section{Visibility as a Locational Factor in the Megaliths of Southern Galicia}

\author{
Miguel Carrero-Pazos \\ Institute of Archaeology. University College London. 31-34 Gordon Square. London WC1H OPY, UK. Correo-e: \\ miguel.carrero.pazos@gmail.com.ID ORCID: https://orcid.org/0000-0001-9203-9954
}

Recepción: 7/01/2021; Revisión: 10/02/2021; Aceptación: 30/03/2021

\begin{abstract}
Resumen: Los estudios de la visibilidad de monumentos pretéritos cuentan con larga tradición en el campo arqueológico, siendo una de las aplicaciones de los métodos SIG más importantes en la actualidad. Este trabajo analiza la visibilidad como factor locacional en las concentraciones megalíticas gallegas de Monte Penide y Serra do Galińeiro, utilizando los Sistemas de Información Geográfica y la estadística espacial. La investigación tiene por objetivo analizar si fue la visibilidad hacia los monumentos, desde ellos, o una explicación conjunta, el elemento que determinó el valor espacial de dichas estructuras megalíticas. Para ello, se analiza el territorio geográfico en el que se sitúan los megalitos -visualscapes- y las líneas de horizonte; se realiza un estudio del tamańo de sus cuencas visuales y otro combinado entre las rutas de tránsito natural y la visibilidad de los túmulos. Los resultados apuntan a una complejidad locacional de dicha variable que, con elevada probabilidad, fue crucial a la hora de escoger el emplazamiento de los túmulos megalíticos. Se defiende la idea de que una presencia visual homogénea del túmulo megalítico pudo haber sido uno de los elementos que marcaron la conformación de estructuras espaciales en las comunidades neolíticas, es decir, sus territorios.

Palabras clave: Sistemas de Información Geográfica; estadística espacial; Arqueología del Paisaje; tránsito natural; fenómeno megalítico; Monte Penide; túmulo.
\end{abstract}

AвSTRACT: The analysis of the visual role of ancient monuments has a long tradition in the archaeological field, being one of the most important applications of GIs methods today. This paper analyses the visibility as a locational factor in the megaliths of Monte Penide and Serra do Galineiro, using Geographic Information Systems and spatial statistics. The research aims to analyse if it was the view from the mounds, the view to them, or a joint explanation among both factors, the element which determined the spatial value of the megalithic structures. To this end, the geographic territory in which the megaliths are located-visualscapes-and the horizon lining of the landscape is analysed; a study of the size of the visual basins of the megaliths and the visibility from the natural transit routes is also carried out. The results point out the complexity of this locational variable which, with high probability, was crucial in the choice of the location of the megalithic barrows. We argue that a homogeneous visual presence of the megalithic mound may have been one of the elements that marked the conformation of spatial structures in Neolithic communities, i.e. their territories.

Key words: Geographic Information Systems; Spatial Statistics; Landscape Archaeology; Natural Transit; Megalithic phenomenon; Monte Penide; mound.

Ediciones Universidad de Salamanca / @®®० 


\section{Introducción ${ }^{1}$ : la visibilidad de los megalitos como factor locacional. ¿Todavía un debate abierto?}

Como elementos positivos en el terreno, los monumentos megalíticos son, por definición, estructuras visibles en el territorio ${ }^{2}$. El estudio de las características visuales de estas arquitecturas ha sido un tema relevante en la discusión arqueológica de las últimas décadas, sobre todo en lo concerniente a las Islas Británicas, Francia, España o los Países Bajos. Las interpretaciones aportadas por diferentes investigadores han sido variadas, abarcando desde sentidos territoriales hasta ceremoniales (Fleming, 1971: 155; Thomas, 1993; Bogucki, 1999: 286).

En la actualidad es ya un tópico asumido que los megalitos contaban con funciones allende de las funerarias (al respecto $c f$. Fleming, 1973; Delibes, 1991), un hecho que ha sido entendido en términos de posesión comunal de la tierra (Saxe, 1970; Hanks, 2008: 262), simbolismo (Chapman, 1981) o evidencias de demarcación territorial, con unas tumbas funerarias que buscarían marcar o 'controlar' partes específicas de los paisajes neolíticos (Renfrew, 1976; Thrane, 1998: 275; Martinón-Torres, 2001; Lagerås, 2002; Tilley, 2004: 197; Last, 2007). Otros investigadores hacen hincapié en la existencia de paisajes cosmológicos (Bourgeois,

Este trabajo fue diseńado durante una estancia de investigación en la Facultad de Arqueología de la Univ. de Leiden (Países Bajos), realizada bajo la supervisión del Dr. Q. Bourgeois y gracias a la financiación proporcionada por el plan postdoctoral I2C de la Xunta de Galicia (Modalidad A, 2018), y redactado y finalizado al amparo del programa de investigación e innovación Horizonte 2020 de la Unión Europea, acuerdo de subvención Marie Sklodowska-Curie número 886793. La base de datos utilizada procede del proyecto de investigación "Arqueoloxía e ecoloxía do fenómeno tumular e megalítico no Sur de Galicia (1998-2000)” (IP: A. A. Rodríguez Casal), financiado por la Xunta de Galicia. Queremos mostrar agradecimiento a los dos revisores anónimos, cuyas sugerencias han permitido enriquecer este texto. Cualquier error es entera responsabilidad del autor.

2 En este artículo utilizaremos como sinónimos los términos de monumentos megalíticos, túmulos megalíticos y megalitos, sin distinción de si existe, o no, cámara funeraria o corredor.
2013), en los que los elementos topográficos y la presencia de otros monumentos desempeñarían papeles fundamentales en la preservación de la tradición, convirtiendo a los megalitos en puntos de referencia de paisajes culturales socio-humanizados (Criado y Villoch, 2000; Lagerås, 2002: 188; Cummings y Whittle, 2004: 82).

Si bien no se puede negar que la visibilidad fue un factor crucial en la construcción y función de estos monumentos neolíticos, sea esta positiva o negativa ( $c f$. al respecto López Romero y Walid Sbeinati, 2005) y en lo que se refiere a un sentido ceremonial y funcional (Roese, 1980; Wheatley, 1995), en los trabajos arqueológicos existe una carencia en su contrastación formal, en comparación, por ejemplo, con las investigaciones en torno a túmulos de la Edad del Bronce (Bourgeois, 2013; De Reu et al., 2011; Fischer et al., 1997). En este sentido, los enfoques modernos basados en estadística espacial asistidos por los Sistemas de Información Geográfica pueden proporcionar un claro avance. Así, esta investigación parte de las interpretaciones que pueden extraerse de la literatura arqueológica al uso (De Reu et al., 2011; Bourgeois, 2013: 105): 1. La visibilidad desde los monumentos. Los túmulos megalíticos fueron construidos para ser vistos, por lo que la visibilidad desde ellos tuvo que ser un criterio importante al escoger su emplazamiento. Pese a que existan excepciones que nos aproximan a fenómenos u estrategias de ocultación (López Romero y Walid Sbeinati, 2005), la localización de los megalitos suele relacionarse con funciones territoriales de demarcación, señalización de fronteras naturales, zonas de paso u orientación espacial hacia partes específicas del paisaje, como zonas de laboreo agrícola o el mar (Fischer et al., 1997; Lagerås, 2002; Cummings y Whittle, 2004: 82; Tilley, 2004).

2. Visibilidad hacia el monumento. La presencia inmanente del túmulo en el paisaje permite entender también la existencia de estrategias dispares de visibilización, entendiendo estas como diferentes criterios de localización que coadyuvan a la visibilidad del túmulo en el paisaje. La localización de estos monumentos en la proximidad de 
rutas de tránsito natural y de movimiento de animales parece ser una de las más claras (Renfrew, 1976; Woodward y Woodward, 1996: 277; De Reu et al., 2011; Murrieta-Flores, 2012) $)^{3}$.

3. Visibilidad hacia otros monumentos y elementos naturales del paisaje. La existencia de patrones de intervisibilidad entre monumentos y grupos, elementos señeros del paisaje, la construcción de redes visuales o procesos de estructuración dentro de las necrópolis son aspectos largamente conocidos (por ejemplo, Wheatley, 1995; Woodward, 2000; Tilley, 2004). Esto puede observarse, por ejemplo, en la relación espacial y visual existente entre túmulos megalíticos y petroglifos u elementos naturales de los paisajes como afloramientos rocosos, que pudieron haber desempeñado un papel de señalización ritual en lugares de arraigada tradición funeraria (Criado y Vaquero, 1991; Criado y Villoch, 2000).

4. Visibilidad en relación con la conectividad y el movimiento por el paisaje: las aproximaciones descritas en los tres puntos anteriores fueron estudiadas en relación a los patrones de movimiento del paisaje, concluyendo la existencia de relaciones tanto cuantitativas (proximidad) como cualitativas -conexión visual-entre los túmulos megalíticos y corredores naturales de paso, rutas tradicionales o caminos de trashumancia, aspectos que han recibido especial atención por parte de la investigación española (Criado y Vaquero, 1991; Eguileta, 1999, 2000; García Sanjuán et al., 2006; Murrieta-Flores, 2012) .

\section{2. Área de estudio, datos arqueológicos e investigaciones previas}

Este trabajo centra su atención en una zona específica del sur de Galicia perteneciente a las comarcas de Vigo y el Baixo Miño (Fig. 1), en la que tanto

3 Also cf. Murrieta-Flores, P.: Travelling through past landscapes. Analysing the dynamics of movement during Late Prehistory in Southern Iberia with spatial technologies. PhD Dissertation defended in 2012 in the Univ. of Southampton.

4 Murrieta-Flores, op. cit. n. 3. los trabajos de prospección arqueológica llevados a cabo por el equipo de A. Rodríguez Casal como el Servicio de Arqueoloxía de la Xunta de Galicia, catalogaron más de un centenar de túmulos megalíticos.

La zona de interés definida en la Figura 1, que denominamos Monte Penide y Serra do Galiñeiro ${ }^{5}$ por ser estos los conjuntos montańosos que mayor cantidad de monumentos albergan, ya ha sido objeto de investigaciones previas. En concreto, suelen citarse las investigaciones de Álvarez Limeses (1935), Mergelina (1936), Díaz Álvarez (1973) o Filgueira Valverde y García Alén (1977), así como Hidalgo Cuñarro y Costas Goberna (1979). En momentos más actuales tenemos los trabajos de Gómez Nistal (Gómez Nistal y Rodríguez Casal, 2000), como parte de su tesis de licenciatura, o el del Fábregas Valcarce (2010), que, versando sobre arte rupestre, trata parte del fenómeno megalítico de la zona.

En términos arqueológicos, la zona de estudio se compone de varios conjuntos megalíticos que, en número total, superan la centena. En concreto, hablamos de los conjuntos megalíticos de Alveadosa, Monte Penide, As Pereiras, As Xunqueiras, Chan de Padrado, Cotogrande, Monte Penide y Monte Vixiador, por citar aquellos más numerosos, que suman una muestra total de 121 monumentos. Pertenecen, todos ellos, a la comarca de Vigo y parte del Baixo Miño, los actuales ayuntamientos de Soutomaior, Pazos de Borbén, Redondela, Mos, Vigo, O Porriño, Tui, Gondomar y Nigrán.

Desde el punto de vista arquitectónico, tenemos desde cámaras funerarias que conservan estructuras ortostáticas hasta túmulos de tierra sin aparentes estructuras pétreas interiores. Necrópolis como la de Cotogrande, en Vigo, han mostrado, tras excavaciones arqueológicas, una importante variabilidad en fórmulas constructivas, con megalitos que han sufrido transformaciones, modificaciones y reutilizaciones durante milenios, poniendo de relieve

5 Carrero-Pazos, M. (2017): El fenómeno tumular y megalitico en Galicia. Aportaciones desde los Sistemas de Información Geográfica y la Estadística Espacial para el estudio de los patrones de localización. Tesis doctoral presentada en 2017 en la Univ. de Santiago de Compostela. http://hdl. handle.net/10347/15823 
biografías complejas a la vez que interesantes (Abad, 1995: 13). En lo que respecta a la cronología de los conjuntos citados, su consideración es difícil, habida cuenta del reducido número de dataciones existentes
(Gómez Nistal, 2001). Excluyendo aquellas que poseen desviaciones estándar elevadas, podemos destacar las obtenidas en Cotogrande 1 (GrN-17698: $5230 \pm 80$ вр; GrN-18420: $4940 \pm 80$ вр), con unos

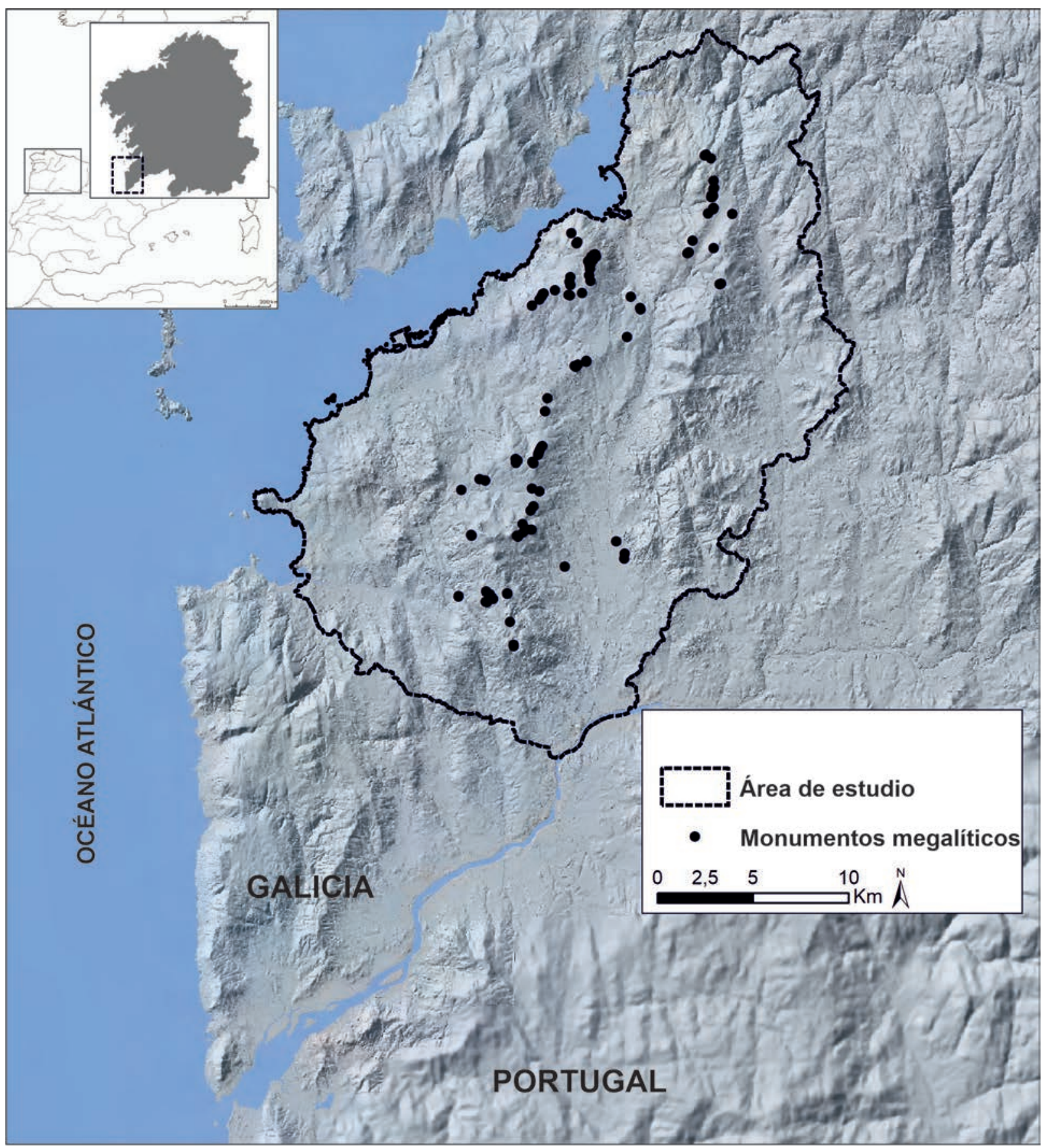

Fig. 1. El área de estudio seleccionada, en el sur de Galicia (No de la Península Ibérica). 


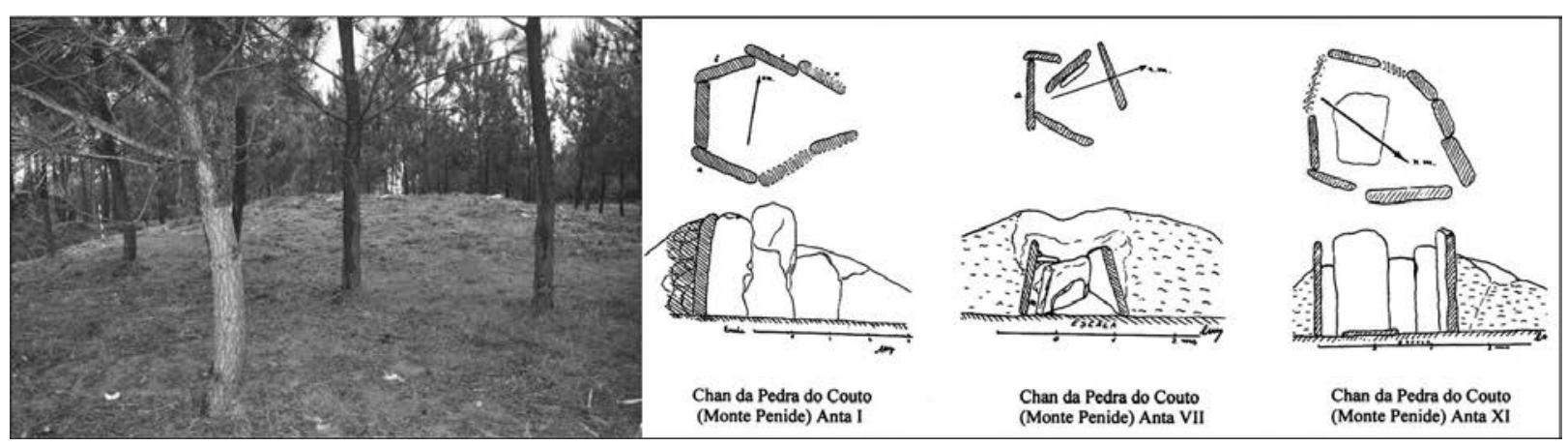

Fig. 2. Fotografia actual de uno de los túmulos megaliticos de Monte Penide y plantas de las excavaciones llevadas a cabo por Mergelina (1936).

márgenes calibrados que nos sitúan entre el 42563937 cal AC para la primera y 3952-3627 cal AC para la segunda (2 sigma, IntCal 20); Cotogrande 2 (GrN-19374: $4875 \pm 40$ вр), 3715-3625 cal АC; Cotogrande 5 (GrN-19565: $4065 \pm 45$ вр; GrN19566: $4390 \pm 50$ вр), 2701-2471 cal АС у 3110$2899 \mathrm{cal}$ AC, respectivamente. Todas ellas muestran una ocupación temporal amplia, con sepulturas utilizadas durante varios milenios, en lo que parece constituir, siguiendo a Fábregas y Vilaseco (2015), un 'cementerio sempiterno' (Fig. 2).

Los conjuntos monumentales estudiados aquí han sido visitados en campo por el citado equipo de investigación -hoy grupo de investigación GePN-Aat, de la Univ. de Santiago de Compostela- y comprobados en una segunda fase por nosotros mismos mediante métodos de groundtruthing, a través del tratamiento de datos Lidar. Esto nos ha permitido solucionar errores de precisión en las coordenadas UTM.

En lo que respecta a los objetivos de este artículo, destacamos a continuación los trabajos de los últimos años, sobre todo aquellos derivados de la aplicación de los SIg y la estadística espacial en Galicia. Estos trabajos suponen una masa crítica para este estudio, a la vez que son una continuación en la discusión de los patrones locacionales del fenómeno megalítico gallego, sumándose a una ya extensa bibliografía (Eguileta, 1999; Criado y Villoch, 2000; Llobera, 2015; Carrero-Pazos y Rodríguez Casal, 2019; Rodríguez Rellán y Fábregas, 2019). En concreto, podemos señalar que los túmulos megalíticos de Monte Penide y Serra do Galiñeiro se localizan muy próximos a rutas de tránsito naturales que discurren $\mathrm{N}-\mathrm{S}$, potencialmente de larga distancia y que conectan diferentes partes del paisaje. Dicha relación debe entenderse tanto en términos cuantitativos - proximidad territorial- como cualitativos -conexión visual desde las propias rutas y los túmulos megalíticos-. Estas zonas suelen coincidir con aquellas áreas geográficas de ruptura de cuenca hidrográfica, evidenciando que los constructores de megalitos hacían uso explícito de los paisajes pretéritos, que, desde el momento de la construcción, quedaban remodelados. Del estudio de los volúmenes tumulares y su contrastación con análisis clúster se ha podido concluir la existencia de una estructura inherente a los diferentes conjuntos (véase Carrero-Pazos et al., 2019), existiendo lo que parece ser un ordenamiento del tipo satelital (Eogan, 1997), con agrupaciones de monumentos de reducido tamaño pero que coinciden con la existencia de grandes túmulos individuales. Dicha disposición parece repetirse en todas las necrópolis de la zona y no es el resultado del azar, desentrañando, quizás, la existencia de una organización subyacente en la composición de estas necrópolis, que podría tener una explicación cultural o religiosa. Esta aseveración es, no obstante, de carácter hipotético, dado que no existen en la actualidad dataciones de $\mathrm{C}^{14}$ que nos permitan analizar las tendencias cronológicas o la evolución de todos estos conjuntos, aspectos clave para entender su funcionamiento estructural. 


\section{Métodos y resultados}

\subsection{Muestreo de base y pruebas de Monte Carlo}

Las preferencias locacionales y las intenciones humanas son cuestiones que pueden ser potencialmente modeladas y formalizadas con el uso de un sig y la estadística espacial, a través de la creación de modelos teóricos y su contrastación con los datos arqueológicos (Jones, 2006; Verhagen y Whitley, 2012) ${ }^{6}$. En lo concerniente a las preferencias locacionales, los investigadores normalmente utilizan aproximaciones comparativas entre la localización de los yacimientos arqueológicos y una población de base -background population, en inglés-, que puede estar creada bajo condiciones aleatorias o regulares sobre la totalidad del área de estudio (Wheatley, 1995; Lake y Woodman, 2000; Conolly y Lake, 2006; García Sanjuán et al., 2006). No obstante, las implicaciones prácticas de esta aproximación tienden a provocar una pérdida en su poder de significación cuando el área de estudio es muy amplia, dado que la comparación de la localización específica de ciertos yacimientos con lugares muy distantes producirá, casi con total seguridad, un resultado estadístico significativo, que por extensión indicará que ambas muestras son diferentes y, por lo tanto, que la localización de los sitios arqueológicos es específica y atiende a ciertas variables - para esta discusión, véase Lake y Woodman, 2000; Verhagen y Whitley, 2012-.

En aras de solucionar esta problemática, a menudo desatendida en los estudios arqueológicos, algunos autores han introducido planteamientos de mejora interesantes, como optar por áreas geográficas similares a la hora de construir las muestras aleatorias (véase, al respecto, Smith y Cochrane, 2011; Bourgeois, 2013). Sin embargo, existen dos

6 Para el desarrollo de este trabajo se han utilizado los softwares ArcGIS 10.4, GRASS GIS 7.4.1 (GRASs Development Team, 2017), Quantum GIS 2.18.14 (QGIs Develpoment Team, 2019) y r Statistics ( $\mathrm{R}$ Development Core Team, 2011), en especial el paquete spatstat (Baddeley y Turner, 2005; Baddeley et al., 2015). cuestiones que es necesario plantearse. La primera se concreta en cómo definir e identificar aquellas zonas del paisaje que realmente pueden compararse con las que poseen, en nuestro caso, túmulos megalíticos y, por extensión, qué variables deben seleccionarse y por qué estas y no otras. Si bien en algunos contextos puede ser perfectamente aceptable tomar todo un paisaje determinado como elemento comparativo, incluso con varios kilómetros de extensión, en Galicia esto se convierte en una cuestión de suma relevancia, ya que estamos ante una región caracterizada por fuertes contrastes geográficos, con montañas y fuertes pendientes que nos llevan a pequeñas llanuras y valles en cuestión de unos pocos kilómetros. En segundo lugar, y tal como ha señalado recientemente Lewis (2020), los enfoques habituales no tienen en cuenta las dinámicas de interacción entre los propios monumentos - denominados, en términos estadísticos, 'factores de segundo orden'- al analizar, por ejemplo, la visibilidad de los sitios, por lo que la estructura de la interacción espacial de los megalitos en relación con sus propiedades visuales es, a menudo, olvidada.

Este trabajo intenta avanzar en los planteamientos metodológicos habituales a través de la comparación de la tendencia de los monumentos megalíticos con dos tipos de muestras aleatorias: una primera, que tiene en cuenta la totalidad del área de estudio -muestreo aleatorio de base-, y otra que constriñe la construcción de la muestra de datos aleatoria a ciertas partes del paisaje, en concreto, aquellas zonas con valores predictivos elevados para los conjuntos megalíticos estudiados -muestreo constreñido-. La primera se utilizará para dibujar un esquema de la totalidad del paisaje en el que se encuentran los monumentos, mientras que la segunda podrá evidenciar la existencia de preferencias locacionales específicas, de existir. Para ello, utilizamos los resultados de trabajos previos que nosotros mismos hemos realizado (Carrero-Pazos, 2018a: 545), partiendo de un modelo locacional que ha sistematizado, mediante el uso de los SIG y la estadística espacial, aquellas variables manejadas por los 


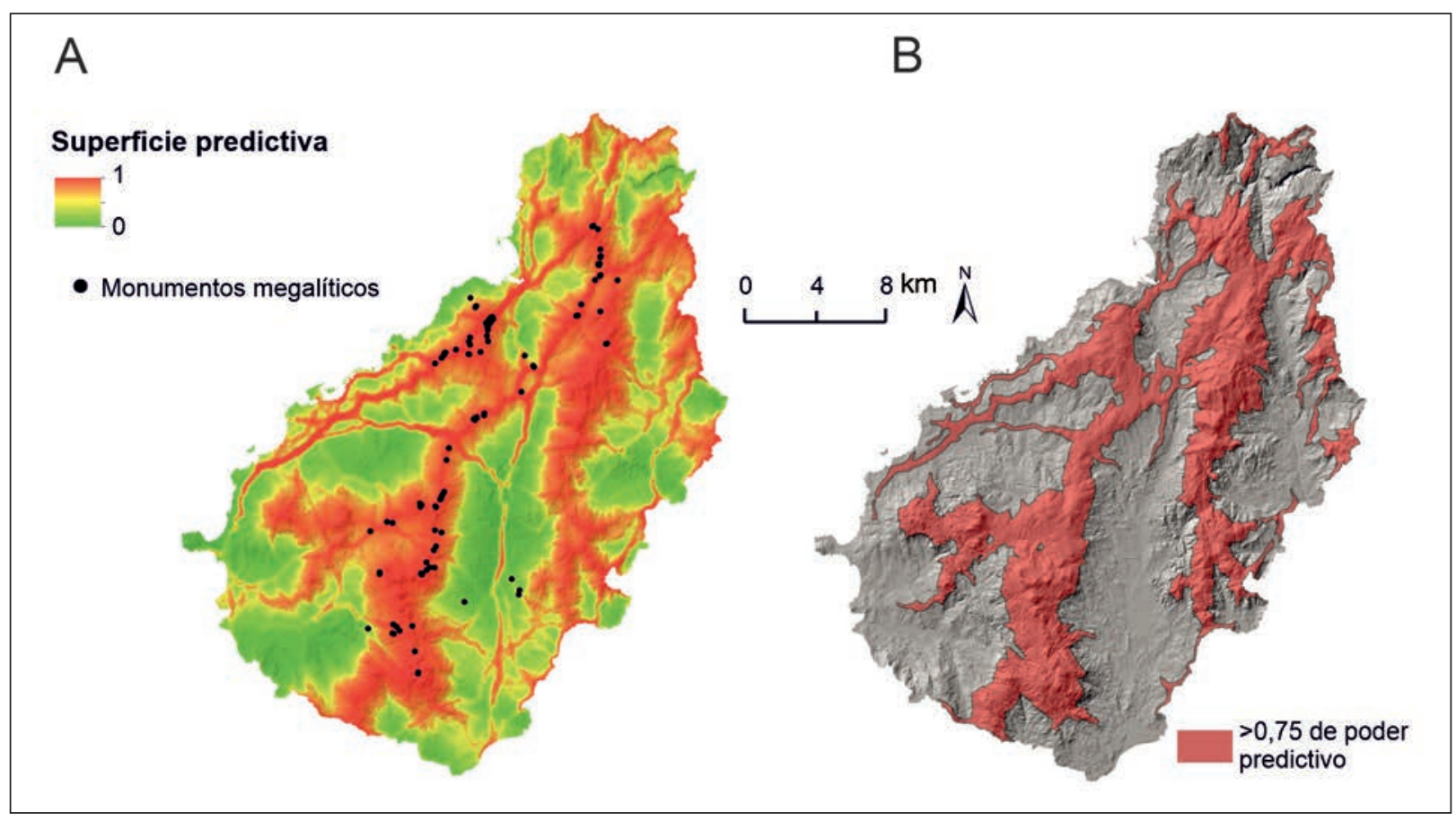

FIG. 3. Superficie predictiva obtenida de Carrero-Pazos, 2018a: 545 (A) y áreas del paisaje constreñidas a valores altos de predicción (>0,75), sobre un sombreado de relieve (B).

investigadores en la bibliografía al uso. Para crear el modelo predictivo de esta zona se utilizó un modelo probabilístico, que señala las partes del paisaje que comparten las regularidades espaciales de los megalitos (Fig. 3A). De esta manera, se obtuvieron conclusiones acerca de la selección de ciertos territorios geográficos y las decisiones locacionales que fueron tomadas para construir los megalitos, basadas en diferentes variables que predecían la distribución de los monumentos: la altitud, la densidad de rutas de tránsito naturales, la red fluvial potencial y el coste del tránsito desde zonas de acumulación potencial de agua.

Por lo tanto, la superficie predictiva obtenida se utilizó para obtener las áreas del paisaje de Monte Penide y Serra do Galiñeiro que poseen valores altos de predicción de túmulos megalíticos $(>0,75)$, lo que nos permite crear una muestra de base de tendencia restringida para este trabajo (Fig. 3B).

Para testar y analizar preferencias de ubicación específicas utilizaremos el método estadístico de
Monte Carlo de simulaciones aleatorias condicionales. Ambas poblaciones estadísticas definidas anteriormente se estudiarán utilizando un conjunto de 999 simulaciones aleatorias compuestas, a su vez, por un número de casos igual al número de túmulos megalíticos -121-. Por lo tanto, la tendencia espacial de los diferentes monumentos será comparada con dos enfoques aleatorios, compuestos en su totalidad por 120.879 puntos -999*121-. Los valores resultantes de estas simulaciones se combinarán con los valores observados de los túmulos, utilizando aquellos clasificados desde la posición número 25 y 975 para definir los límites de un rango aleatorio, que encierra el $95 \%$ de los conjuntos de datos aleatorios, a fin de proporcionar una prueba de significación al $5 \%$ -0,05-. Este método no ha sido desarrollado originalmente por nosotros, sino que se trata de una adaptación de los trabajos de Bevan y Lake (véase al respecto Bevan et al., 2013: 49; Baddeley et al., 2015: 46; Bevan, 2020). 


\subsection{Monumentos en el paisaje. ¿Fueron los monumentos megalíticos construidos en localizaciones con elevada visibilidad?}

La visibilidad es, quizás, el criterio de localización más citado y repetido en los trabajos sobre el Megalitismo gallego (Criado, 1988; Eguileta, 1999). La pregunta que buscamos responder es la siguiente: ¿están los monumentos megalíticos de Monte Penide y Serra do Galiñeiro localizados en las zonas del paisaje más visibles? Para responderla, utilizamos varios procesos metodológicos que implican el uso de un sig y la estadística espacial.

El primer enfoque realizado se refiere a la creación de un mapa de visibilidad total -total viewshed-, tal y como lo describe Llobera (Llobera et al., 2010), en el que se calcula la visibilidad desde todas las celdas que componen un Modelo Digital de Elevaciones -en adelante MDE-. Este proceso analítico produce un mapa que expresa la prominencia visual del paisaje, identificando aquellas zonas que, de forma natural, son más y menos visibles. La segunda aproximación que hemos utilizado es el factor de vista de cielo -Sky View Factor, en adelante SVF-, que proporciona una medida de la apertura del terreno (Zakšek et al., 2011), y se utiliza aquí para comprobar si los monumentos megalíticos se encuentran emplazados en zonas limitadas por el relieve circundante o poseen panorámicas de extenso cielo abierto. Además, hemos complementado estos análisis con un estudio del patrón monovariante a través de la 'intensidad' de los monumentos con respecto a cada una de estas variables. Se trata de calcular el número esperado de puntos por unidad de área si la cantidad de los mismos fuese homogénea en toda la zona, con el objetivo de observar si la distribución de los megalitos tiende a concentrarse en medidas particulares o no (Bevan, 2020). Esto puede realizarse mediante un resumen no paramétrico de cada relación univariante entre la presencia de monumentos megalíticos y ambas covariables, implementado en el software $R$ Statistics utilizando la función r.hohat del paquete spatstat (Baddeley et al., 2015: 180).
El mapa de visibilidad total fue creado a partir de un MDE de $2 \mathrm{~m}$ de resolución obtenido a partir de datos LidAR y descargado del Centro Nacional de Información Geográfica -CNIG-, bajo licencia CC-BY 4.0. Este modelo ha sido creado a partir de un proceso de filtraje de datos, considerando solo los puntos del terreno, con lo que se han substraído los elementos artificiales como edificios. Además, se le aplicó un suavizado de decimación para obtener un paso de malla final de $5 \mathrm{~m}$. Esto permite contar con una resolución suficiente para los cálculos al tiempo que minimiza, en los procesos analíticos, la interferencia de vallas, taludes de carreteras o caminos.

Este MDE fue convertido a puntos $-\mathrm{n}=$ 24.783.875- y para cada punto se calculó la visibilidad acumulativa. Este análisis fue realizado en Quantum GIs 2.18.14, utilizando la herramienta de visibilidad avanzada (Čučković, 2016) ${ }^{7}$.

Tal y como se observa en la Fig. 4A, el resultado de ambas aproximaciones nos permite concluir que los monumentos megalíticos no se localizan en las zonas más visibles del paisaje, aunque sí parecen localizarse en posiciones visibles si consideramos escalas locales. Desde esta perspectiva, los resultados de la simulación de Monte Carlo señalan que la visibilidad podría estar actuando como una variable determinante en la localización de los megalitos, ya que estos muestran una tendencia específica que difiere de ambas muestras aleatorias (Fig. 4B). Esta conclusión está en clara sintonía con los resultados obtenidos para otras partes de Galicia, como A Serra do Barbanza (Bustelo et al., 2018; Carrero-Pazos et al., 2020).

Por otra parte, tal y como se describe en Zakšek et al., 2011, el factor de vista del cielo -SVF- proporciona un valor de la porción de cielo visible para cada celda, limitado por el relieve circundante. El SVF fue calculado en GRASS GIS 7.4.1 con el módulo r.skyview, y los valores del ráster resultantes abarcan desde 1, que identifica las superficies terrestres no obstruidas -normalmente las superficies horizontales-, y 0, para aquellas superficies que se encuentran

7 http://zoran-cuckovic.from.hr/QGIS-visibility-analysis/ 


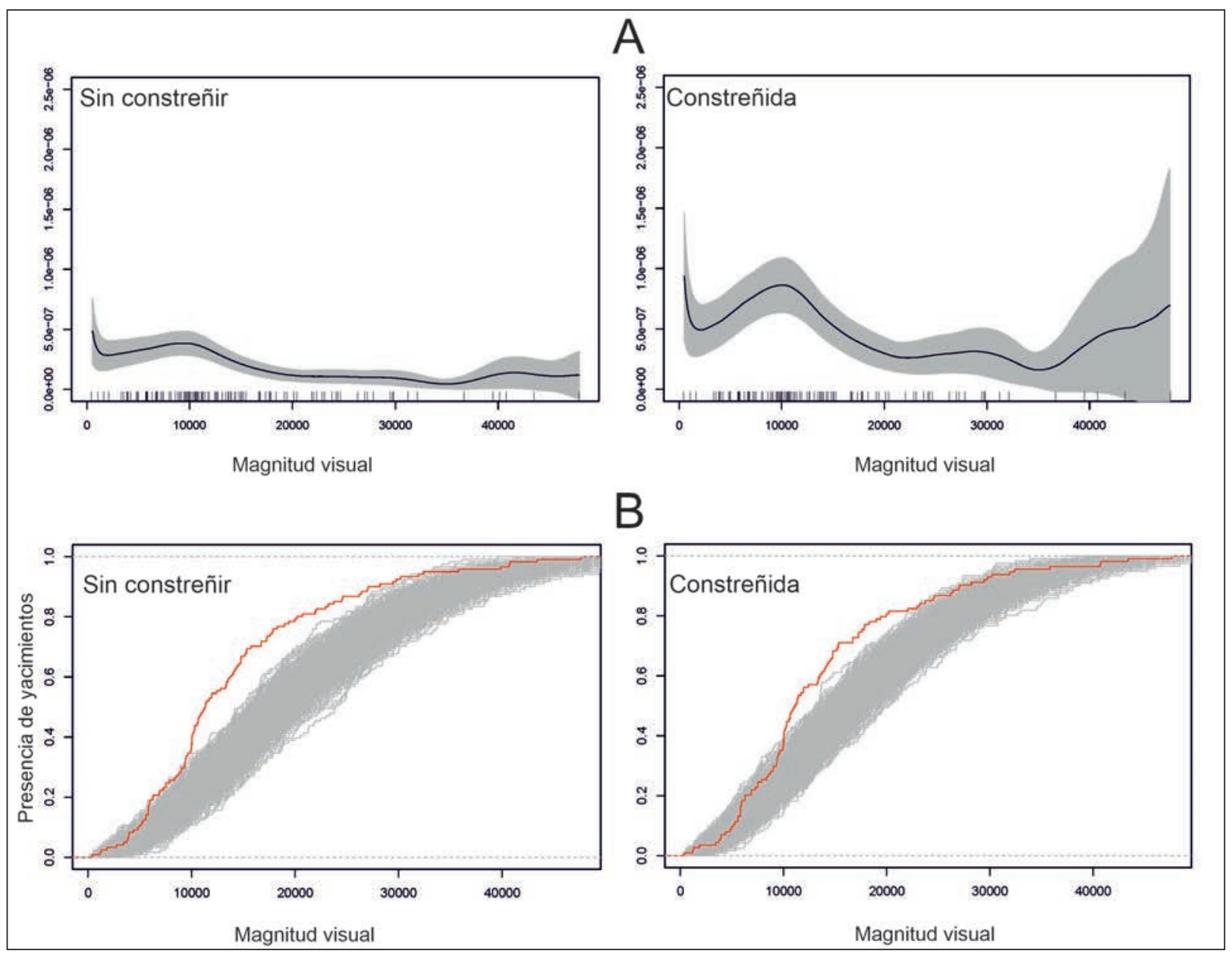

FIG. 4. Aproximaciones metodológicas para el estudio de la magnitud visual y su comparación con muestreos aleatorios de base - muestra sin constreñir/muestra constreñida-: A) densidad de monumentos como una función de la magnitud visual-las líneas negras sólidas muestran la estimación de la función mientras que el sombreado gris se corresponde con un intervalo aleatorio del $95 \%$ de significación-; B) simulación de Monte Carlo (la línea identifica la tendencia de los monumentos, mientras que el rango gris encierra el $95 \%$ del conjunto de datos aleatorio).

completamente obstruidas, cuya vista del cielo es inexistente o está bloqueada geográficamente.

Los resultados del patrón monovariante indican que los túmulos se encuentran localizados en áreas del paisaje abiertas (Fig. 5A), una característica que es más visible si comparamos la tendencia mostrada por los monumentos con la mostrada por la muestra aleatoria constreñida. Por su parte, la simulación de Monte Carlo muestra también esta divergencia que parece tornarse, ahora, en preferencial, al no coincidir la tendencia mostrada por los túmulos con la de ambas muestras aleatorias (Fig. 5B).

\subsection{La visibilidad desde el monumento. ¿Fue la visibilidad desde los túmulos algo importante?}

Para continuar entendiendo el rol visual que los monumentos megalíticos de Monte Penide y Serra do Galiñeiro pudieron haber desempeñado, estudiamos ahora las cuencas visuales de los monumentos, y un enfoque interesante que podemos aplicar se basa en observar las regularidades de las cuencas visuales de los megalitos -en la línea de lo planteado por Lake y Woodman (2000)-. El objetivo es observar si el tamaño de la cuenca visual de los 


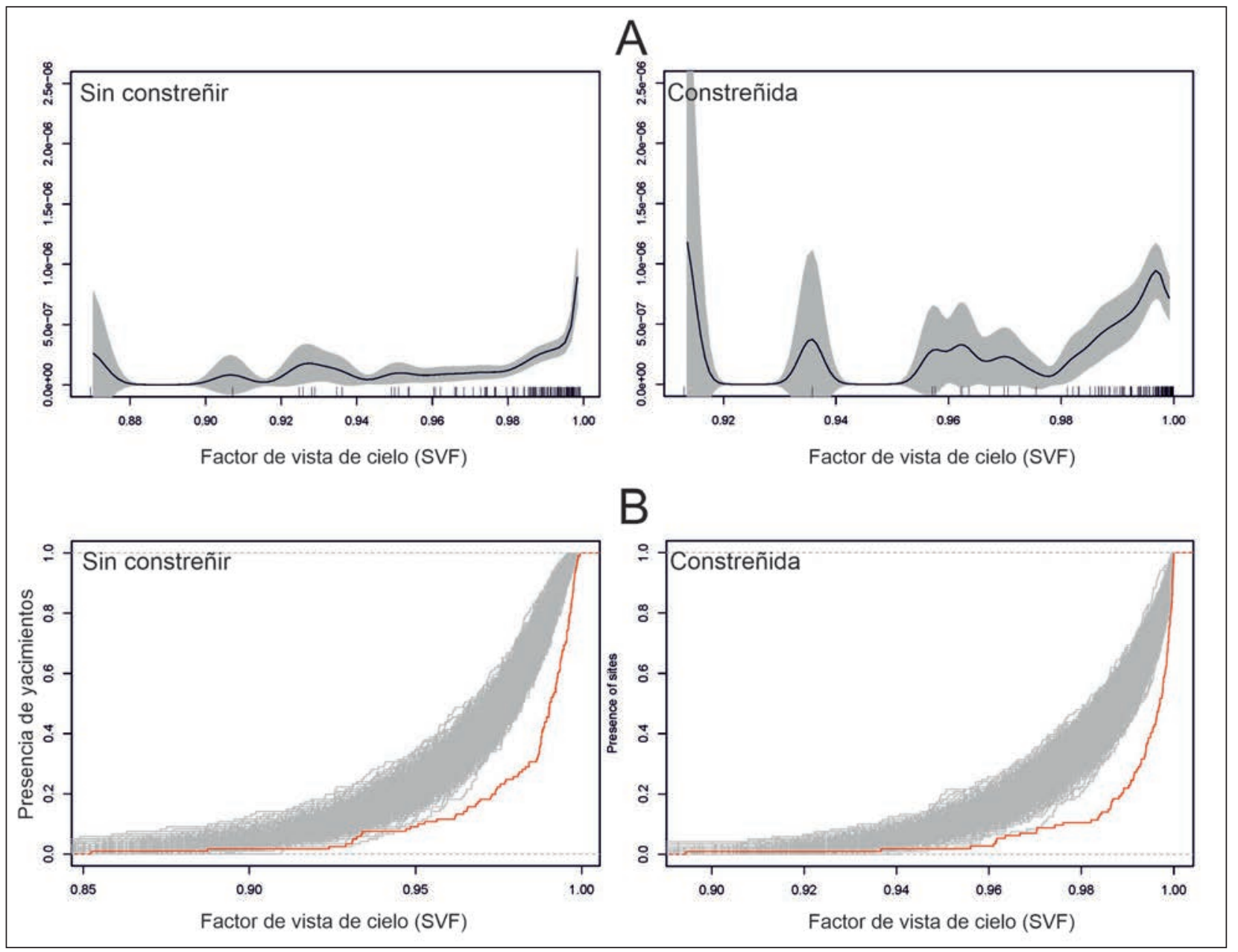

FIG. 5. Aproximaciones para el estudio del factor de vista de cielo o Skyview Factor de los monumentos megaliticos y su comparación con muestreos aleatorios de base - muestra sin constreñir/muestra constreñida-: A) densidad de monumentos como una función del factor de vista de cielo -las lineas sólidas muestran la estimación de la función, mientras que el sombreado gris se corresponde con un intervalo aleatorio del $95 \%$ de significación-; B) simulación de Monte Carlo-la línea identifica la tendencia de los monumentos, mientras que el rango gris encierra el $95 \%$ del conjunto de datos aleatorio-.

megalitos está conectado con el paisaje o si difiere, indicando, de esta forma, que pudo haber existido algún tipo de elección específica teniendo en cuenta el tamaño de los campos visuales de los monumentos. Tal y como venimos realizando, se utilizaron dos poblaciones de base para esta comparación, una referida a toda el área de estudio y otra constreñida a aquellas zonas con valores espaciales similares a los de los túmulos megalíticos.

Para ello, se calculó la visibilidad acumulativa tanto para los túmulos como para ambas muestras aleatorias, utilizando GRASS GIS 7.4.1. ${ }^{8}$, con r.stats para obtener el área de la cuenca visual de cada punto - en $\mathrm{km}^{2}-$. Los resultados pueden analizarse visualmente mediante distribuciones de proporciones acumuladas (Fig. 6), pudiendo observar que la línea negra-monumentos megalíticos- cae enteramente

8 El tamaño de la cuenca visual de todas las muestras fue calculado sobre los resultados del análisis de visibilidad total, con un radio de $500 \mathrm{~m}$ y una elevación del observador de 1,65 m, con lo que se redujeron, sustancialmente, los tiempos de procesado. 


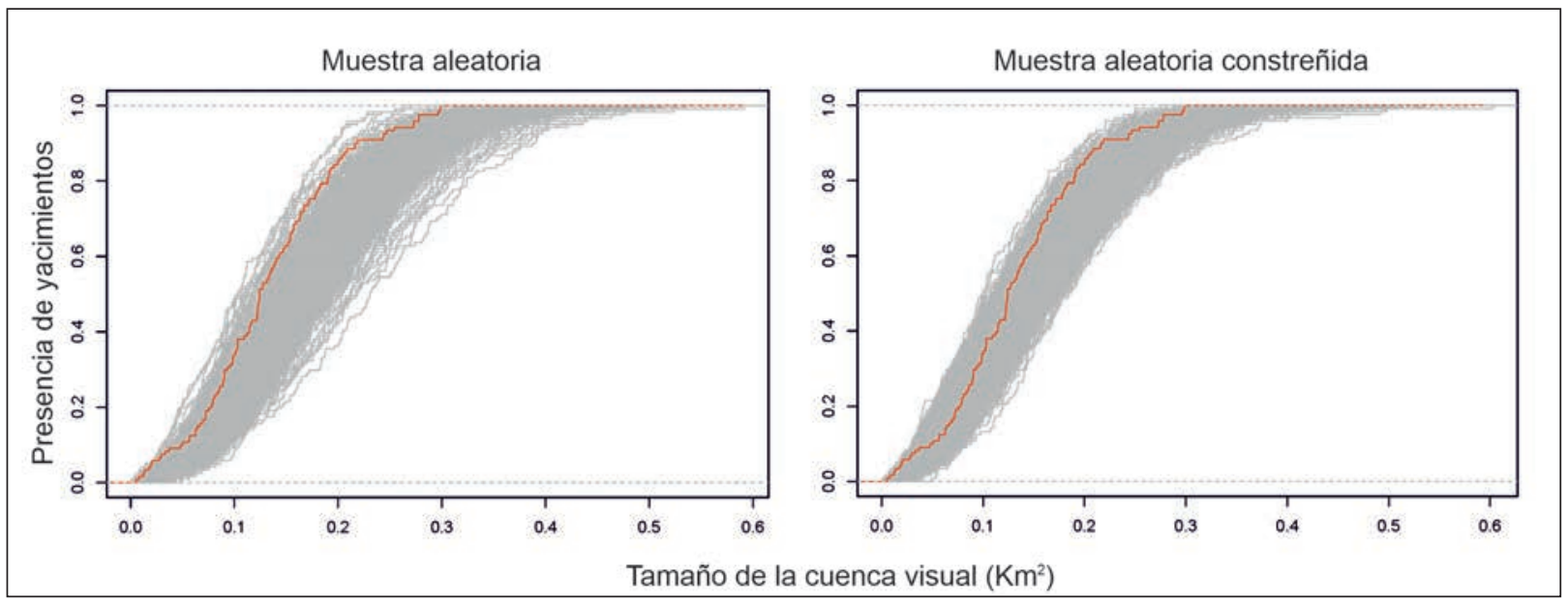

FIG. 6. Distribución de proporciones acumuladas del tamaño de la cuenca visual de los monumentos megalíticos y ambas muestras aleatorias.

en el rango gris aleatorio. Esto nos permite concluir que el tamaño de la cuenca visual de los monumentos no es una variable significativa, ya que existen similares probabilidades de obtener idénticos tamaños de cuenca visual por los túmulos megalíticos que por puntos obtenidos al azar. Sin embargo, hay tendencias específicas en las cuencas visuales medianas que parecen diferir de las muestras de base, ya que la línea negra sobre los $0,2 \mathrm{~km}^{2}$ está muy cerca del límite superior del rango gris de muestras aleatorias.

\subsection{La visibilidad hacia los megalitos o los megalitos que estructuran el paisaje}

La relación de los monumentos megalíticos con el movimiento por el paisaje es un hecho bien investigado en zonas como el sur peninsular o diferentes áreas europeas, territorios en los que diferentes investigadores han propuesto que dichas estructuras deben ser entendidas como elementos que estructuran y remodelan los paisajes neolíticos. En este trabajo seguimos las investigaciones de Murrieta-Flores (2012) ${ }^{9}$ y Llobera (2015) para modelizar con sig y estadística espacial el movimiento natural por el paisaje. En segundo lugar, se estudiarán las

9 Murrieta-Flores, op. cit. n. 3. propiedades visuales de los sitios megalíticos en relación con las rutas generadas.

Este tipo de aproximación debe ser entendido ante todo como un modelo teórico, pues no pretende de ninguna forma reconstruir las rutas prehistóricas -cf. Verhagen et al., 2019, para una revisión completa de los métodos más utilizados, y Llobera, 2020, para las últimas aproximaciones-.

Partimos de la creación de un modelo general de movilidad humana, a partir de la suma de múltiples rutas de menor coste - least cost path- calculadas sobre el MDE del área de estudio. El objetivo es obtener un mapa de densidad de rutas que mostrará aquellas áreas del paisaje que atraen mayor o menor movimiento de forma natural. En términos técnicos, el proceso se ha descrito en otro lugar (Carrero-Pazos, 2018b), aunque señalaremos ahora que se han creado un total de 209.306 rutas de menor coste, que fueron sumadas en un ráster resultante, tal y como apreciamos en la Fig. 7A.

Por lo tanto, los túmulos megalíticos se localizan muy próximos a las rutas, en lo que parece ser puntos de paso a lo largo de redes de comunicación a escala local y regional. Posteriormente calculamos la relación visual de los megalitos con dicho tránsito, a través de un cálculo de visibilidad acumulativa. Los resultados (Fig. 7B) indican las áreas del paisaje que pueden ser vistas desde los megalitos, por lo que 
podemos seleccionar aquellos valores para las rutas de tránsito y observar qué partes mantienen una conexión visual elevada. Tal y como se observa, los túmulos son más visibles desde las rutas que ascienden y descienden de las sierras, hecho que probablemente indique un uso de estos monumentos como marcadores territoriales locales, aunque el grado de dicha relación no sea absoluto y, por norma general, las agrupaciones norteñas sean más visibles que las del sur.

Otra aproximación que podemos explorar es el nivel de perceptibilidad (o identificación) de los túmulos en el paisaje. En estas situaciones, el contraste del objeto de estudio con el fondo es crucial para reconocer el elemento - $c f$. Fábrega-Álvarez y Parcero-Oubińa, 2019, para una propuesta novedosa con respecto a la visibilidad de individuos-, y existen diferentes análisis que pueden usarse para estudiar este aspecto, como la localización de un túmulo megalítico en una posición de cielo abierto para aumentar su sensación de monumentalidad.

Siguiendo los trabajos de Bourgeois (2013), en este artículo utilizamos un estudio del skyline o línea del horizonte, calculada desde una posición determinada - punto de observador-. Tal y como venimos realizando, se creó una muestra de base con 999 simulaciones de 121 observadores y el mapa producido fue comparado con la tendencia mostrada por los túmulos megalíticos, resultando que los monumentos se localizan, en general, sobre áreas de elevada concentración de líneas de horizonte, que acostumbran a coincidir con las crestas montañosas (Fig. 8).

Por otra parte, para saber si esta relación positiva mostrada con la densidad de puntos del horizonte puede estar relacionada también con el movimiento

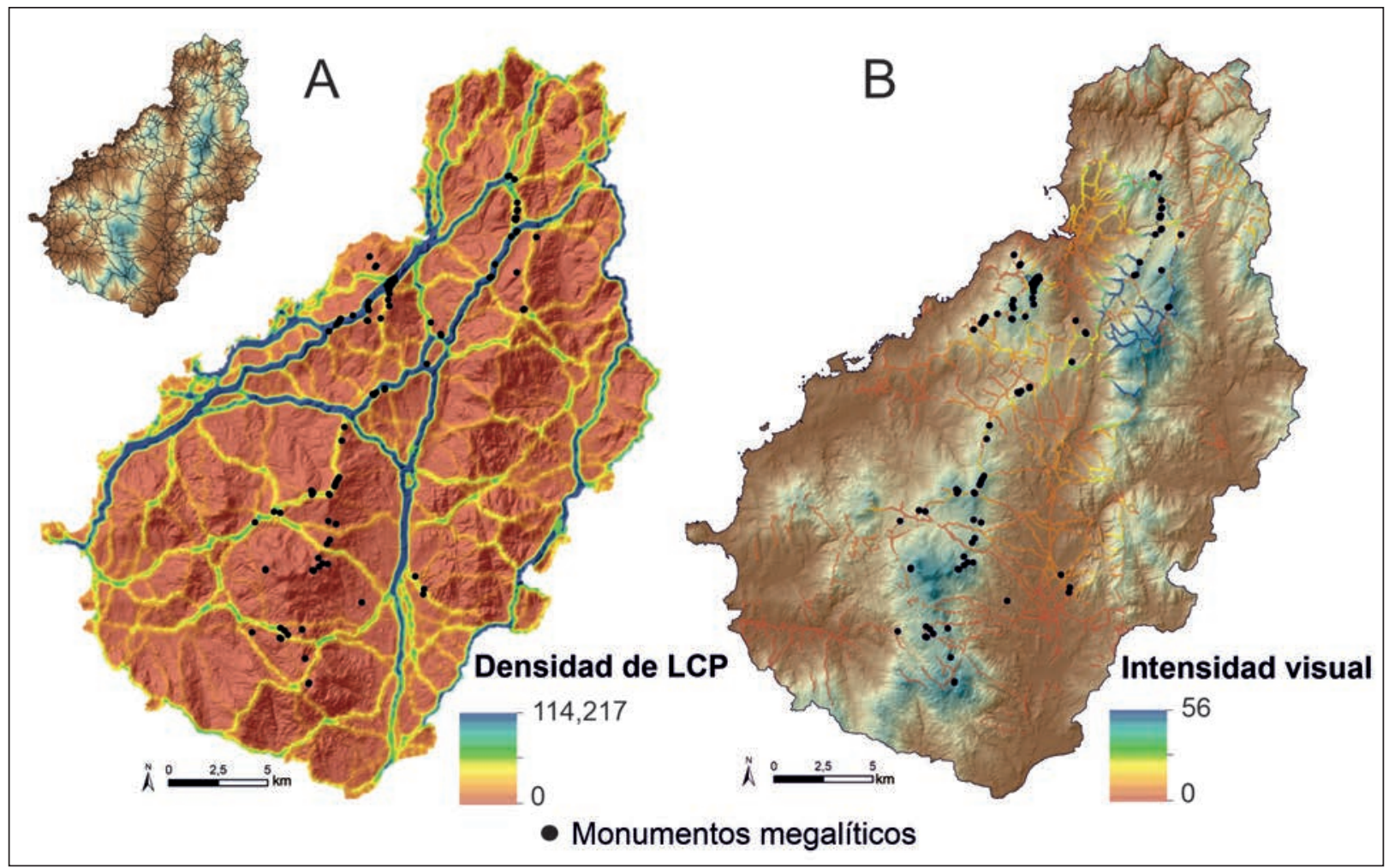

Fig. 7. Relación entre el tránsito natural y la visibilidad de los túmulos megalíticos. A) Intensidad de rutas de tránsito potencial en la región de Monte Penide y Serra do Galiñeiro, con las rutas de menor coste resultantes -least cost paths- en la parte superior; B) Intensidad visual de las rutas de tránsito calculada desde los monumentos megaliticos. 
y estructuración por el paisaje, se calcularon las líneas de horizonte visual para 4 rutas seleccionadas como observadores, tal y como se aprecia en las Figs. 9 y 10. En estos casos, los megalitos están localizados en los límites del horizonte visual calculado desde las rutas de tránsito, construyendo lo que semeja, de esta forma, un paisaje de tipo cultural enmarcado visualmente por la presencia de los monumentos. La excepción viene con la ruta n. ${ }^{\circ}$ 3 (Fig. 10), que cuenta con líneas de horizonte bastante restringidas a su localidad más inmediata.

\section{Discusión}

Las aproximaciones analíticas desarrolladas en las líneas anteriores nos permiten, a continuación,

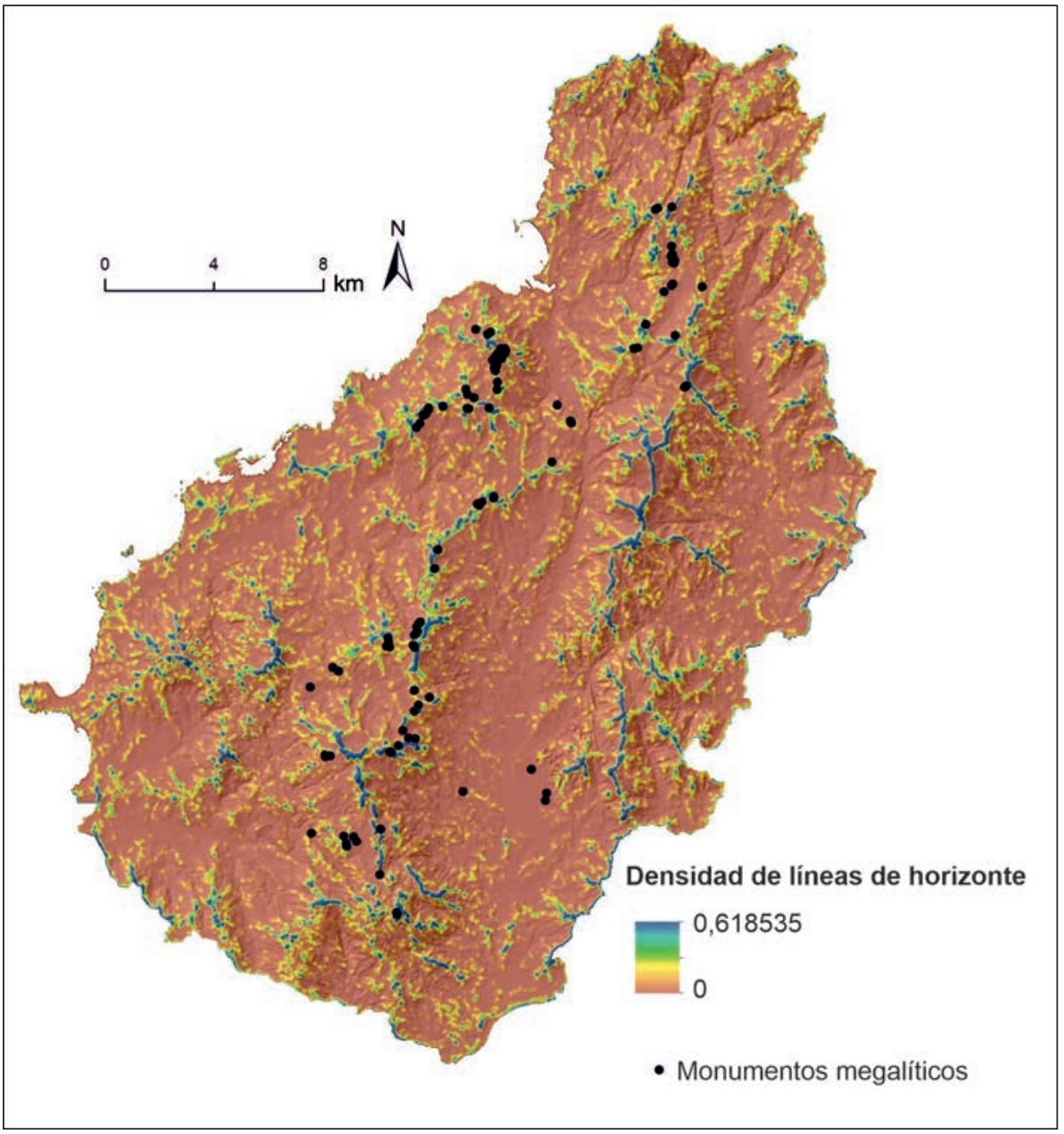

FIG. 8. Densidad de lineas de horizonte. Ráster de densidades Kernel creado a partir de 999 muestras aleatorias de 121 puntos. discutir las cuestiones históricas planteadas al inicio (Bourgeois, 2013: 142).

a) ¿Están los túmulos megalíticos emplazados en zonas de elevada visibilidad?

A tenor de los análisis realizados, los megalitos de Monte Penide y Serra do Galiñeiro no fueron construidos en las zonas de mayor visibilidad del área de estudio. Esta conclusión coincide con los resultados para otras áreas gallegas -cf., por ejemplo, Rodríguez Rellán y Fábregas, 2019), con concentraciones de monumentos ubicadas en zonas de prominencias visuales medias, pero nunca las mayores (Bustelo et al., 2018). Esto se complementa con los resultados comparativos de las simulaciones de Monte
Carlo, que permiten concluir que las tendencias de los megalitos difieren de las muestras de base -tanto la que se corresponde con la totalidad del área de trabajo como aquella constreñida a las zonas de 'tendencia megalítica'-. Por lo tanto, esto nos indica que la visibilidad es un criterio locacional importante: las áreas de magnitudes visuales medias parecen ser aquellas de selección preferencial a la hora de emplazar los monumentos megalíticos. Ello se ve complementado con los resultados del factor vista de cielo -o Sky View Factor-, pues estamos ante espacios de cielo abierto, superficies de tierra sin obstáculos a la vista importantes, un patrón que también ha 


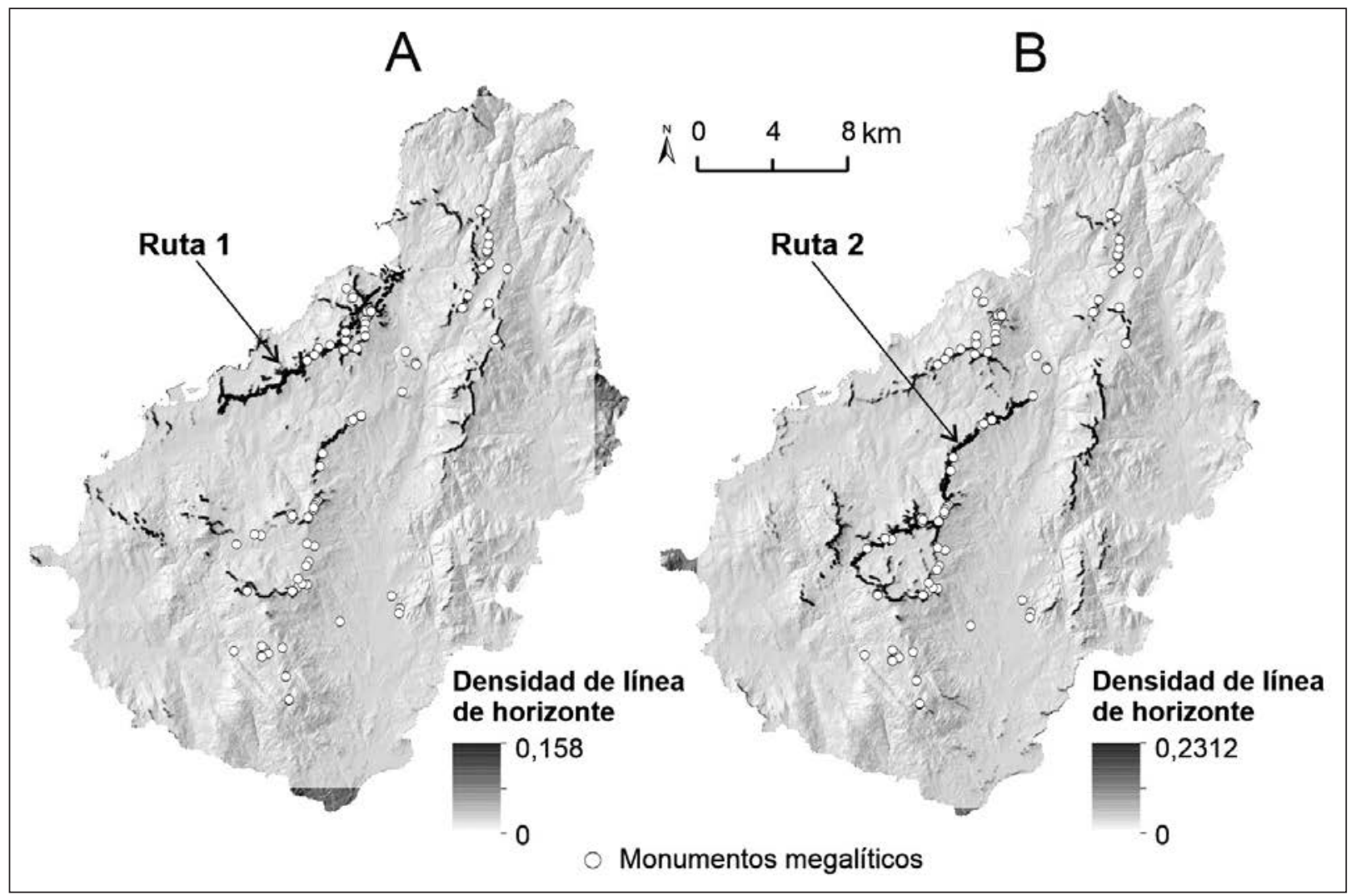

Fig. 9. Linea de horizonte calculada desde dos rutas de tránsito potencial centrales en el paisaje de Monte Penide y Serra do Galiñeiro.

sido detectado en otras zonas (por ejemplo, Bustelo et al., 2018).

b) ¿Fue la visibilidad de los túmulos megalíticos un factor importante a la hora de seleccionar sus ubicaciones particulares?

La respuesta es afirmativa. Los túmulos megalíticos, como estructuras positivas, fueron construidos para ser vistos. Aunque esta respuesta puede ocultar otros procesos interesantes que no han recibido la suficiente atención por parte de los investigadores, como casos de 'ocultación' o 'camuflaje', atestiguados mediante la construcción de pequeños montículos, enterramientos sin estructuras o montículos positivos -por ejemplo, López Romero y Walid Sbeinati, 2005-.

Tomando como base el trabajo de M. Lake y P. Woodman (2000), hemos abordado la cuestión de si la visibilidad es un factor clave en la elección del emplazamiento para las sepulturas megalíticas (entendida ahora como las vistas o panorámicas tomadas desde los túmulos megalíticos como observadores) a partir del estudio del tamaño de sus cuencas visuales. Los resultados nos indican que tanto la muestra arqueológica como aquellas aleatorias tienen una tendencia similar y que los monumentos poseen cuencas visuales de tamaño medio. Si bien esto podría ser el resultado del propio paisaje, consecuencia, por ejemplo, de cambios repentinos en la topografía, cuando consideramos la relación de los megalitos con las redes de tránsito natural, dicha conclusión cambia. La localización de los túmulos megalíticos parece estar en estrecha relación con el movimiento natural por el paisaje, un hecho especialmente evidente en las concentraciones tumulares septentrionales. Esas zonas de movimiento 


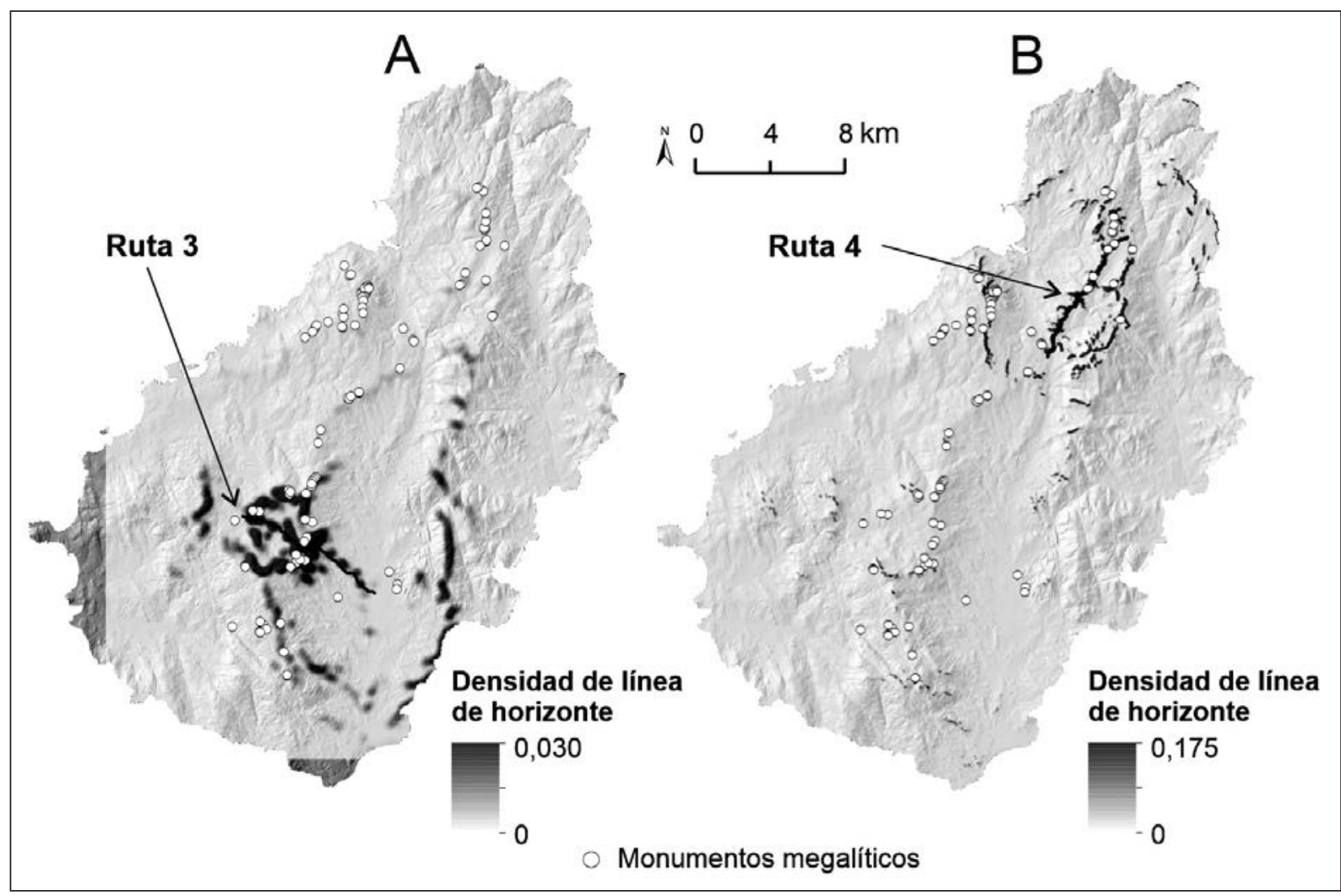

Fig. 10. Linea de horizonte calculada desde dos rutas de tránsito potencial localizadas en los extremos del paisaje de Monte Penide y Serra do Galiñeiro.

coinciden, por su parte, con las crestas y bordes de cuencas hidrográficas que drenan hacia la costa $(\mathrm{Ca}-$ rrero-Pazos et al., 2019).

Otro punto interesante es que los túmulos están situados en zonas de elevada densidad de líneas de horizonte, lo que indica, desde un punto de vista general, que los monumentos son hitos visibles desde su territorio cercano y mantienen su presencia sobre los horizontes visuales de la totalidad del paisaje. De hecho, pueden ofrecerse ulteriores argumentos si se consideran las líneas de horizonte calculadas desde las rutas de tránsito potencial. En tres de los cuatro casos analizados (Figs. 9 y 10), los megalitos están localizados sobre las líneas de horizonte calculadas desde las rutas, lo que, quizás forzando el argumento, puede ser leído en clave de territorialidad, con unos monumentos megalíticos que se configuran como señales permanentes de la posesión del terreno.

\section{Conclusión}

En este artículo se evalúa la importancia de la visibilidad como factor de localización en los megalitos del s de Galicia, utilizando para ello los Sistemas de Información Geográfica y la estadística espacial. Se suma, por tanto, a trabajos anteriores que se ocuparon de estudiar otros factores locacionales, como la asociación de los túmulos y rutas de tránsito natural, y pone de relieve, además, la necesidad de continuar estudiando, con métodos cuantitativos y diseños metodológicos complementarios, variables analizadas en trabajos anteriores. Esta investigación es una continuación del estudio de las variables topográficas expuestas en Carrero-Pazos et al., 2019, mediante la consideración, en mayor profundidad, del significado visual del túmulo en el paisaje, algo no realizado hasta el momento. 
Los análisis desarrollados aquí indican que el factor visual de las concentraciones tumulares y megalíticas de Monte Penide y Serra do Galiñeiro fue un criterio relevante a la hora de elegir la ubicación específica para la construcción de estas estructuras funerarias. La evidencia analítica aportada apunta que fue la visibilidad hacia los megalitos el criterio que pudo determinar su valor espacial en la zona, con monumentos situados en zonas de gran densidad de líneas de horizonte que suelen coincidir con aquellas áreas de ruptura de cuenca hidrográfica. Desde esta perspectiva, entonces, el megalito es entendido como un hito visual en el terreno que, con su presencia inmanente, parece tener una función de demarcación territorial en tiempos neolíticos. Estos resultados ayudan a apoyar, por tanto, los argumentos territoriales de localización expuestos en Carrero-Pazos (2019), investigación que concluye que los monumentos de la zona están localizados, y distribuidos espacialmente, manteniendo un criterio jerárquico que parece caracterizar una territorialidad específica. En este contexto, los análisis propuestos evidencian que es la visibilidad el factor espacial que juega un papel clave en la articulación de los túmulos megalíticos en el paisaje. De hecho, el valor espacial de la visibilidad hacia el túmulo megalítico es una variable que también se registra en otras áreas de Galicia, caso del fenómeno megalítico del Barbanza (Bustelo et al., 2018), u otros territorios peninsulares (García Sanjuán et al., 2006; Cabrero et al., 2020).

Los paisajes megalíticos de Monte Penide y Serra do Galiñeiro se corresponden con espacios abiertos, en los que la visibilidad relacionada con estas estructuras tuvo que haber desempeñado un papel trascendental para las comunidades neolíticas, tal y como indica la conexión visual entre estos monumentos y el movimiento a través del paisaje. Pese a que nos encontremos en un contexto de incertidumbre cronológica, con la inexistencia de dataciones directas para la práctica totalidad de conjuntos estudiados, los resultados del análisis de la línea del horizonte proporcionan claves interesantes sobre el tipo de estructuras sociales que los megalitos materializaban al ser construidos, ya que los

Ediciones Universidad de Salamanca / @@ఠ conjuntos mantienen, por su localización en el paisaje, una presencia visual muy homogénea. Además, sabemos que se encuentran conectados visualmente, en lo que parece expresar un sentido territorial, de posesión de la tierra. Si bien esto es más evidente en los túmulos megalíticos del norte de la región considerada, no ocurre lo mismo en los del sur, que parecen situarse ante paisajes más cerrados, tal vez muestra de otro tipo de fenómenos divergentes con la tendencia general.

\section{Bibliografía}

Abad, X. C. (1995): "Un ejemplo de readaptaciones constructivas en un enterramiento tumular: Cotogrande n. ${ }^{\circ}$ 5", Minius: Revista do Departamento de Historia, Arte e Xeografia, 4, pp. 13-30.

Álvarez Limeses, G. (1935): Geografía del Reino de Galicia. Tomo de la Provincia de Pontevedra. Barcelona: Casa Editorial Alberto Martín.

Baddeley, A. J.; Rubak, E. y Turner, R. (2015): Spatial Point Patterns. Methodology and Applications with $R$. Boca Raton: CrC Press.

Baddeley, A. J. y Turner, R. (2005): "Spatstat: an R package for analysing spatial point patterns", Journal of Statistical Software, 12 (6), pp. 1-41.

Bevan, A. (2020): "Spatial point pattern and processes". En Gillings, M., Hacigüzeller, P. y Lock, G. (eds.): Archaeological Spatial Analysis. A Methodological Guide. New York: Routledge, pp. 60-76.

Bevan, A.; Crema, E.; Xiuzhen, L. y Palmisano, A. (2013): "Intensities, interactions and uncertainties: some new approaches to archaeological distributions". En Bevan, A. y Lake, M. (eds.): Computational approaches to archaeological landscapes. Walnut Creek, California: Left Coast Press, pp. 27-52.

Bogucki, P. (1999): The origins of human society. Malden: Blackwell.

Bourgeois, Q. (2013): Monuments on the Horizon. The formation of the barrow landscape throughout the 3rd and 2 nd millennium BC. Leiden: Sidestone Press.

Bustelo, J.; Rodríguez Rellán, C.; Fábregas, R. y Barbeito, V. (2018): "Alén da Serra. O fenómeno tumular na Península do Barbanza a través dos six e a estatística espacial", Gallaecia, 36, pp. 53-72.

Cabrero, C.; Cámara, J. A. y Esquivel, J. A. (2020): "Patrones de distribución espacial en las necrópolis 
megalíticas de Majadillas y Hoyas del Conquín (Granada, España). Resultados preliminares", Lucentum, xxxix, pp. 73-85.

Carrero-Pazos, M. (2018a): "Beyond the scale. Building formal approaches for the study of spatial patterns in Galician moundscapes (Nw Iberian Peninsula)", Journal of Archaeological Science: Reports, 19 , pp. 538-551. https://doi.org/10.1016/j.jasrep.2018.03.026

Carrero-Pazos, M. (2018b): "Modelando dinámicas de movilidad y visibilidad en los paisajes megalíticos gallegos. El caso del Monte de Santa Mariña y su entorno (Comarca de Sarria, Lugo)", Trabajos de Prehistoria, 75 (2), pp. 287-306. https://doi. org/10.3989/tp.2018.12216

Carrero-Pazos, M.; Bevan, A. y Lake, M. (2019): "The spatial structure of Galician megalithic landscapes: a case study from the Monte Penide region", Journal of Archaeological Science, 108, https://doi.org/10.1016/j.jas.2019.05.004

Carrero-Pazos, M.; Bustelo-Abuín, J.; Barbeito-PoSE, V. y Rodríguez Rellán, C. (2020): "Locational preferences and spatial arrangement in the barrow landscape of Serra do Barbanza (North-western Iberia)", Journal of Archaeological Science: Reports, 31, https://doi.org/10.1016/j.jasrep.2020.102351

Carrero-Pazos, M. y Rodríguez Casal, A. A. (2019): "General and local spatial trends in Galician megalithic landscapes”. En Hinz, M.; MülLer, J. y Wunderlich, M. (eds.): Megaliths, Societies and Landscapes: Early Monumentality and Social Differentiation in Neolithic Europe. Bonn: Habelt, pp. 641665.

Chapman, R. (1981): "Archaeological theory and communal burial in prehistoric Europe”. En Hodder, I.; Isaac, G. y Hammond, N. (eds.): Pattern to Process. Studies in Honour of David Clarke. Cambridge: CuP, pp. 387-412.

Conolly, J. y Lake, M. (2006): Geographic Information Systems in Archaeology. Cambridge: cup.

Criado, F. (1988): "Arqueología del Paisaje y Espacio Megalítico en Galicia. Seminario sobre Arqueología Espacial (Tomar, 1988)", Arqueología Espacial, 12, pp. 61-117.

Criado, F. y Vaquero, J. (1991): "El fenómeno megalítico y tumular: formas diversas de pasado monumental". En Criado, F. (ed.): Arqueología del paisaje. El área Bocelo-Furelos entre los tiempos paleoliticos y medievales. A Coruña: Xunta de Galicia, pp. 129-146.
Criado, F. y Villoch, V. (2000): "Monumentalizing landscape: from present perception to the past meaning of Galician Megalithism (north-west Iberian Peninsula)", European Journal of Archaeology, 3 (2), pp. 188-216.

ČuČKović, Z. (2016): “Advanced viewshed analysis: a Quantum GIs plug-in for the analysis of visual landscapes", The Journal of Open Source Software, 4 (1), 32. doi:10.21105/joss.00032

Cummings, V. y Whittle, A. (2004): Places of special virtue: megaliths in the Neolithic landscapes of Wales. Oxford: Oxbow Books.

Delibes de Castro, G. (1991): "Megalitos ¿Todavía una civilización de muertos?", Arqrítica. Crítica de Arqueología Española, 2 (2), pp. 9-10.

De Reu, J.; Bourgeois, J.; De Smedt, P.; Zwertvaegher, A.; Antrop, M.; Bats, M.; De Maeyer, P.; Finke, P.; Van Meirvenne, M.; Verniers, J. y Crombé, P. (2011): "Measuring the relative topographic position of archaeological sites in the landscape, a case study on the Bronze Age barrows in northwest Belgium”, Journal of Archaeological Science, 38, pp. 3435-3446.

Díaz Álvarez, P. (1973): Noticias de prehistoria en torno a Vigo y su ría. Vigo: Caja de Ahorros Municipal de Vigo.

Eguileta, J. M.a (1999): A Baixa Limia na Prehistoria Recente. Arqueoloxia dunha Paisaxe na Galicia interior. Ourense: Diput. de Ourense.

Eguileta, J. M. a (2000): "Megalitos e Tránsito: Aproximación ó estudio de vías de comunicación prehistóricas", Lethes, 2, pp. 46-61.

EOGan, G. (1997): "Overlays and underlays: aspects of megalithic art succession at Brugh na Bóinne, Ireland”. En Bello, J. M. (ed.): Actas III Coloquio Internacional de Arte Megalítico. Brigantium, 10. A Coruña: Diput. de A Coruña, pp. 217-234.

Fábrega-Álvarez, P. y Parcero-Oubiña, C. (2019): "Now you see me. An assessment of the visual recognition and control of individuals in archaeological landscapes", Journal of Archaeological Science, 104, pp. 56-74.

FÁBREGAs, R. (2010; [2001 ed. orig.]): Os petróglifos e o seu contexto. Vigo: Instituto de Estudios Vigueses.

Fábregas, R. y Vilaseco, X. I. (2015): Building forever or just for the time? En Laporte, L. y SCARre, C. (eds.): The Megalithic Architectures of Europe. Oxford: Oxbow Books, pp. 101-110.

Filgueira, J. y García Alén, A. (1977): “Inventario de monumentos megalíticos de la provincia de 
Pontevedra”, El Museo de Pontevedra, 31, pp. 43130.

Fischer, P. F.; Farrelly, C.; Maddocks, A. y RugGLES, C. L. N. (1997): "Spatial Analysis of visible areas from the Bronze Age cairns of Mull", Journal of Archaeological Science, 24, pp. 581-592.

Fleming, A. (1971): "Territorial patterns in Bronze Age Wessex", Proceedings of the Prehistoric Society, xx, pp. 138-166.

Fleming, A. (1973): “Tombs for the living", Man, 8 (2), pp. 177-193.

García Sanjuán, L.; Metcalfe-Wood, S.; Rivera, T. y Wheatley, D. W. (2006): "Análisis de pautas de visibilidad en la distribución de monumentos megalíticos de Sierra Morena Occidental". En Grau, I. (ed.): La aplicación de los SIG en la Arqueología del Paisaje. Alicante: Univ. de Alicante, pp. 181-200.

Gómez Nistal, C. (2001): "Neolitización y Megalitismo en el entorno de la ría de Vigo: estado actual de la investigación”, Gallaecia, 20, pp. 39-60.

Gómez Nistal, C. y Rodríguez Casal, A. A. (2000): "El fenómeno tumular y megalítico en la Galicia suroccidental: aspectos historiográficos y estado actual de la investigación”. En Jorge, V. O. (ed.):

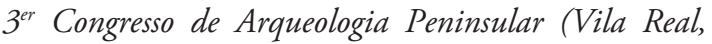
2000)-Neolitizaçao e Megalitismo na Peninsula Iberica. Porto: ADECAP, pp. 321-336.

grass Development Team (2017): Geographic Resources Analysis Support System (GRASS) Software, Version 7.4.1. Open Source Geospatial Foundation. Electronic document: http://grass.osgeo.org

Hanks, B. (2008): "The past in later Prehistory". En Jones, A.: Prehistoric Europe, Theory and Practice. Oxford: Blackwell, pp. 255-284.

Hidalgo, J. M. y Costas, F. J. (1979): "El gran conjunto megalítico de Monte Penide. Redondela-Pontevedra”, El Museo de Pontevedra, 34, pp. 81-93.

Jones, E. E. (2006): "Using Viewshed Analysis to Explore Settlement Choice: A Case Study of the Onondaga", American Antiquity, 71 (3), pp. 523-538.

LAGERÅs, K. E. (2002): "Visible intentions? Viewshed analysis of Bronze Age burial mounds in Western Scania, Sweden". En Scarre, C. (ed.): Monuments and landscape in Atlantic Europe, perception and society during the Neolithic and the Early Bronze Age. London: Routledge, pp. 179-191.

LaKe, M. y Woodman, P. (2000): "Viewshed analysis of site location on Islay". En Mithen, S. J. (ed.): Hunter-gatherer landscape archaeology: the Southern Hebrides Mesolithic Project, 1988-98. Cambridge: The
McDonald Institute for Archaeological Research, pp. 497-503.

LAST, J. (2007): Beyond the grave, new perspectives on barrows. Oxford: Oxbow Books.

LEwIS, J. (2020): "Visibility of the Gask Ridge Road from Simulated Watchtowers: A Monte Carlo Testing Approach", Journal of Archaeological Science: Reports, 33, https://doi.org/10.1016/j.jasrep.2020.102482

Llobera, M. (2015): "Working the Digital: Some thoughts from Landscape Archaeology". En Chapman, R. y Wylie, A. (eds.): Material Evidence: Learning from Archaeological Practice. Abingdon: Routledge, pp. 173-188.

Llobera, M. (2020): "Memory at your feet: Modeling the agency of past trails", Journal of Archaeological Science: Reports, 29, https://doi.org/10.1016/j.jasrep.2019.102177

Llobera, M.; Wheatley, D.; Steele, J.; Cox, S. y Parchment, O. (2010): "Calculating the inherent visual structure of a landscape ('Total Viewshed') using high-throughput computing". En Nicolucci, F. y Hermon, S. (eds.): Beyond the Artefact. Digital Interpretation of the Past. Proceedings of CAA2004 (Prato, 2004). Budapest: Archaeolingua, pp. 146-151.

López Romero, E. y Walid Sbeinati, S. (2005): "Estrategias de ocultación en el megalitismo tumular del centro-oeste peninsular". En Ontañón, R.; GaRCía-Moncó, C. y Arias, P. (eds.): Actas III Congreso del Neolítico en la Peninsula Ibérica (Santander, 2003). Santander: Univ. de Cantabria, pp. 697-704.

Martinón-Torres, M. (2001): Os monumentos megaliticos despois do Megalitismo. Arqueoloxía e Historia dos megalitos a través das fontes escritas (s. VI-S. XIX). Valga: Concello de Valga.

Mergelina, C. (1936): "Notas sobre arqueología gallega. Las mámoas del Chan da Pedra do Couto y de San Colmado", Boletín del Seminario de Estudios de Arte y Arqueología de Valladolid, 10, pp. 1-33.

Murrieta-Flores, P. (2012): “Understanding human movement through spatial technologies: the role of natural areas of transit in the Late Prehistory of South-western Iberia”, Trabajos de Prehistoria, 69 (1), pp. 103-122.

Qgis Development Team (2019): Qgis Geographic Information System. QGIS Association. http://www.qgis. org

R Development Core Team (2011): $R$ : A language and environment for statistical computing (vol. http:// www.R-project.org). Vienna: R Foundation for Statistical Computing. 
Renfrew, C. (1976): "Megaliths, territories and populations". En De Laet, S. L. (ed.): Acculturation and Continuity in Atlantic Europe, mainly during the Neolithic period and the Bronze Age. Papers Presented at the IV Atlantic Colloquium. Ghent. Bruges: De Tempel, pp. 198-220.

Rodríguez Rellán, C. y Fábregas, R. (2019): “Monuments on the move. Assessing megaliths' interaction with the North-Western Iberian landscapes". En Hinz, M.; Müller, J. y Wunderlich, M. (eds.): Megaliths, Societies and Landscapes: Early Monumentality and Social Differentiation in Neolithic Europe. Bonn: Habelt, pp. 621-639.

Roese, H. (1980): "Some aspects of topographical location of Neolithic and Bronze Age monuments in Wales 1: Menhirs", Bulletin of the Board of Celtic Studies, 28, pp. 645-655.

SAXE, A. A. (1970): Social dimensions of mortuary practices. Michigan: Univ. of Michigan.

Smith, C. y Cochrane, E. E. (2011): "How is visibility important for defence? A GIS analysis of sites in 361 the Western Fijian Islands", Archaeology in Oceania, 46, pp. 76-84. https://doi. org/10.1002/j.1834-4453.2011.tb00101.x

Thomas, J. (1993): "The politics of vision and the archaeologies of landscape". En Bender, B. (ed.): Landscape: Politics and Perspectives. Oxford: Berg, pp. 19-48.

Thrane, H. (1998): "The effects of the Bronze Age on the environment and culture in Scandinavia". En
Hänsel, B. (ed.): Mensch und Umwelt in der Bronzezeit Europas. Kiel: Oetker-Voges, pp. 271-280.

Tilley, C. (2004): The Materiality of Stone. Explorations in Landscape Phenomenology. Oxford: Berg.

Verhagen, P.; Nuninger, L. y Groenhuijzen, M. R. (2019): "Modelling of Pathways and Movement Networks in Archaeology: An Overview of $\mathrm{Cu}-$ rrent Approaches". En Verhagen, P.; Joyce J. y Groenhuijzen M. (eds.): Finding the Limits of the Limes. Cham: Springer, pp. 217-249. https://doi. org/10.1007/978-3-030-04576-0_11

Verhagen, P. y Whitley, T. G. (2012): "Integrating Archaeological Theory and Predictive Modeling: a Live Report from the Scene", Journal of Archaeological Method and Theory, 19, pp. 49-100. https://doi. org/10.1007/s10816-0121-9102-7

Wheatley, D. (1995): "Cumulative viewshed analysis: a GIs-based method for investigating intervisibility, and its archaeological application". En Lock, G. y Stancic, Z. (eds.): Archaeology and Geographical Information Systems: an European perspective. London: Taylor and Francis, pp. 171-186.

Woodward, A. (2000): British Barrows, a matter of life and death. Stroud: Tempus Publishing.

Woodward, A. B. y Woodward, P. J. (1996): “The topography of some Barrow Cemeteries in Bronze Age Wessex", Proceedings of the Prehistoric Society, 62, pp. 275-291.

ZAKŠEK, K.; Ostir, K. y KoKalJ, Z. (2011): "Sky-View Factor as a Relief Visualization Technique", Remote Sensing, 3 (2), pp. 398-415. 